\title{
EDITORIAL
}

\section{Multidisciplinary and interdisciplinary research}

The prevalence of multi-author research papers in evidence today is a reflection not only of the fact that most scientists today work in teams, but also of the emergence of multidisciplinary and interdisciplinary research. While it is possible to describe what the terms multidisciplinary and interdisciplinary mean, it is not possible to strictly maintain the distinction between them in actual practice. In fact, the terms are often used interchangeably.

Multidisciplinary research is research involving more than one discipline, where researchers from each discipline make separate contributions towards the research objective, which cannot be achieved within a single discipline. On the other hand, interdisciplinary research involves researchers from different disciplines attempting to integrate concepts and methods from their disciplines to arrive at new insights. It is clear that any research involving researchers from different disciplines will lie somewhere on the continuum between 'pure' multidisciplinary research and 'true' interdisciplinary research. The greater the communication and interaction between researchers coming from different disciplines, the more the research effort will shift towards interdisciplinarity. While multidisciplinary research can help in solving complex problems, it is interdisciplinary research that can lead to creative expansion of the frontiers of science.

Interdisciplinary research can lead to the development of new areas of scientific investigations, which with time take on the characteristics of a single discipline, having its own set of fundamental concepts and knowledge structure. Thus, if one considers Physics, Chemistry and Biology as the basic and fundamental disciplines of science, then Biochemistry and Nanomaterials stand as examples of long established and more recently established disciplines respectively, arising from interdisciplinary efforts. Scientists who enjoy the challenge of interdisciplinary research must of necessity, learn as much as possible of the disciplines they interact with outside their own specialization, and must (in the sense described by the Nobel prize winning biochemist, Albert Szent-Györgyi) be more Dionysian than Apollonian in spirit. 